PROCEEDINGS OF THE

AMERICAN MATHEMATICAL SOCIETY

Volume 139, Number 1, January 2011, Pages 139-152

S 0002-9939(2010)10467-7

Article electronically published on June 29, 2010

\title{
NEAREST NEIGHBOUR DISTANCE AND DIMENSION OF INTENSITY MEASURE OF POISSON POINT PROCESS
}

\author{
RADOSŁAW WIECZOREK
}

(Communicated by Edward C. Waymire)

\begin{abstract}
We prove that the upper and lower local dimensions of a finite measure $\mu$ are equal to the upper and lower limit of $-\log \alpha / \log \hat{r}_{\alpha \mu}(x)$, where $\hat{r}_{\alpha \mu}(x)$ is the mean distance to the closest point for the Poisson point processes with intensity measure $\alpha \mu$. Moreover the upper local dimension of $\mu$ is a.e. bounded from above by the limit superior of $-\log \alpha / \log \hat{r}_{\alpha \mu}$, where $\hat{r}_{\alpha \mu}$ denotes the expected nearest-neighbour distance.
\end{abstract}

In 1954 P.J. Clark and F.C. Evans [5] introduced the measure of spatial relationship in a population, now called the Clark-Evans index or nearest-neighbour index. The index is given by the quotient of observed mean distance to a nearest neighbour divided by the expected value of the mean nearest-neighbour distance of the homogeneous Poisson process. Namely $C E=\hat{r}_{o b s} / \hat{r}$ with

$$
\hat{r}_{o b s}=\frac{1}{N} \sum_{i=1}^{N} \min _{j \neq i} \operatorname{dist}\left(x_{i}, x_{j}\right)
$$

where the $x_{i}$ are positions of observed points, while $\hat{r}$ is, roughly speaking, the expected value of (0.1) if the $x_{i}$ are distributed according to a Poisson point process with Lebesgue intensity measure. On the plane the value of $\hat{r}$ is $\frac{1}{2 \sqrt{\rho}}$, where $\rho$ is the density, i.e. the average number of points per area. The Clark-Evans index is commonly used in the statistical analysis of patterns in ecology, geology, etc., to describe the spatial structure of point patterns; $c f$. [13, 11, 20]. Namely, for randomly, independently and uniformly distributed points we have $C E \approx 1$, while $C E>1$ means a regular structure and $C E<1$ indicates that the points form clusters or aggregative structure.

One can expect that for points distributed according to a fractal measure the Clark-Evans index should be less than one or, looking from another direction, the value of $\hat{r}$ will be different if we replace the Lebesgue measure by a fractal measure. The aim of this paper is, roughly speaking, to investigate how $\hat{r}$ depends on the

Received by the editors October 5, 2009 and, in revised form, November 6, 2009, February 6, 2010 and February 18, 2010.

2010 Mathematics Subject Classification. Primary 28A80; Secondary 60G55.

Key words and phrases. Pointwise dimension, nearest-neighbour distance, measure, Poisson point processes.

This research was partially supported by the State Committee for Scientific Research (Poland) Grant No. N N201 021133 and by EC FP6 Marie Curie ToK programme SPADE2, MTKD-CT2004-014508 and Polish MNiSW SPB-M.

(C)2010 American Mathematical Society Reverts to public domain 28 years from publication 
density if points are distributed according to a Poisson point process with an arbitrary intensity measure. Precisely, for a finite measure $\mu$ and a positive number $\alpha$ and for a point $x \in \operatorname{supp} \mu$, let $\hat{r}_{\alpha \mu}(x)$ denote an expected value of the distance to the point closest to $x$ for a Poisson point process with intensity measure $\alpha \mu$. Then $\hat{r}_{\alpha \mu}(x)$ behaves like $\alpha^{-1 / d_{\mu}(x)}$ for large $\alpha$, where $d_{\mu}(x)$ is the local dimension of the measure $\mu$ at $x$ (Theorem 1.5). If moreover $\hat{r}_{\alpha \mu, q}$ denotes the expected value of the mean nearest-neighbour distance (0.1) to the power $q$, then the upper and lower limits of $-q \log \alpha / \log \hat{r}_{\alpha \mu, q}$ constitute new dimension functions, denoted here by $\operatorname{dim}_{B P}(\mu, q)$, which can be alternatively calculated in the following way: let $\rho_{x}(\beta)$ denote the radius of a ball centered at $x$ and containing the $\beta$-part of the measure $\mu$ and take a limit of $q \log \beta / \log \int \rho_{x}(\beta)^{q} \mu(\mathrm{d} x)$. The latter formula is a convenient alternative for the definition of the dimension function proposed by Badii and Politi in [2]. Badii and Politi studied a dimension function similar to $\operatorname{dim}_{B P}(\mu, q)$ and postulated its relation to the Rényi dimension spectrum. They did not provide any proof besides the calculation on examples. We prove here the mathematically strict version of this relation; see Theorem 2.2. Moreover we check that the upper local dimension of $\mu$ is a.e. bounded from above by the limit superior of $-\log \alpha / \log \hat{r}_{\alpha \mu}$ (Theorem 1.7). It should be stressed that the results are obtained for an arbitrary Borel measure on a metric space.

The result may be used to estimate the dimension of the measure from empirical data. Termonia and Alexandrowicz in 21] exploited the idea of estimating fractal dimension by means of distances to nearest points sampled from the attractor. The idea was developed also in [2] and [3, where the dimension $D$ of the measure was claimed to be the exponent in the relation $\hat{r}(n)=K n^{-1 / D}$, with $n$ being the number of points randomly chosen from the attractor, but proofs were rather heuristic there and referred only to some special measures. However, the authors provided there a very interesting relationship of the defined dimension function and the Rényi dimension spectrum. Some mathematically strict facts are given also in [6]; a result similar to Theorem 1.5 was obtained there, although it covers only measures on $\mathbb{R}^{n}$ and was formulated in a different framework.

\section{MAin RESUlts}

Let $\mu$ be a finite Borel measure on a metric space ( $X$, dist). For the sake of simplicity let us assume that $\mu$ is probabilistic. For $x \in X$ and $r>0$ let us denote:

$$
\begin{aligned}
& B_{x}(r):=\{x \in X: \operatorname{dist}(x, y)<r\}, \\
& \bar{B}_{x}(r):=\{x \in X: \operatorname{dist}(x, y) \leq r\} .
\end{aligned}
$$

Consider a counting measure $N$ (i.e. the measure with integer values only; see e.g. [4) and for an $x \in X$ let $\underline{r}(x, N)$ denote the distance to the closest point, namely:

$$
\underline{r}(x, N):=\sup \left\{r \geq 0: N\left(B_{x}(r)\right)=0\right\}
$$

with $\sup \emptyset=0$ and $\inf \emptyset=\infty$. If we write $N=\sum_{i} \delta_{x_{i}}$ (which we always can), then

$$
\underline{r}(x, N):=\min _{i} \operatorname{dist}\left(x, x_{i}\right) .
$$

From now on let $N_{\alpha \mu}$ be a Poisson point process with intensity measure (or mean measure) $\alpha \mu$, where $\alpha$ is some positive constant. For extensive information on Poisson point processes, see e.g. [14, 7]. We write $\left\|N_{\alpha \mu}\right\|$ for the total number of points, i.e. $\left\|N_{\alpha \mu}\right\|=N_{\alpha \mu}(X)$. We let $\operatorname{Prob}_{\alpha \mu}$ denote the probability distribution 
of the process $N_{\alpha \mu}$ on the space $\mathcal{N}$ of all integer-valued Borel measures, whilst $\mathrm{E}_{\alpha \mu}$ denotes the expected value under the probability measure Prob $_{\alpha \mu}$. Remember also that, since $\alpha \mu$ is finite, $N_{\alpha \mu}$ is a.s. finite.

Definition 1.1. The mean closest-point distance $\hat{r}_{\alpha \mu}(x)$ to a point $x \in X$ is the expected value of $\underline{r}\left(x, N_{\alpha \mu}\right)$ conditional on there being at least one point; i.e.

$$
\begin{aligned}
\hat{r}_{\alpha \mu}(x) & :=\mathrm{E}_{\alpha \mu}^{\geq 1} \underline{r}\left(x, N_{\alpha \mu}\right)=\int_{\mathcal{N}} \underline{r}(x, N) \operatorname{Prob}_{\alpha \mu}^{\geq 1}(\mathrm{~d} N) \\
& =\int_{\mathcal{N}} \underline{r}(x, N) \operatorname{Prob}_{\alpha \mu}(\mathrm{d} N \mid\|N\| \geq 1) .
\end{aligned}
$$

We also define the mean closest-point distance to the power $q$ :

$$
\hat{r}_{\alpha \mu, q}(x):=\mathrm{E}_{\alpha \mu}^{\geq 1} \underline{r}(x, N)^{q}=\int_{\mathcal{N}} \underline{r}(x, N)^{q} \operatorname{Prob}_{\alpha \mu}(\mathrm{d} N \mid\|N\| \geq 1) .
$$

Remark 1.2. Since $\mu$ is finite, the probability that $\left\|N_{\alpha \mu}\right\|=0$ is nonzero; however in such a case the distance to the closest point has no meaning. That is why we disregard that possibility by using the conditional probability, and we write $\operatorname{Prob}_{\alpha \mu}^{\geq 1}(A)=\operatorname{Prob}_{\alpha \mu}\left(A \mid\left\|N_{\alpha \mu}\right\| \geq 1\right)$. Likewise, we need at least two points to measure the distance between them, so we use also the notation $\operatorname{Prob}_{\alpha \mu}^{\geq 2}(A)=$ $\operatorname{Prob}_{\alpha \mu}\left(A \mid\left\|N_{\alpha \mu}\right\| \geq 2\right)$.

Definition 1.3. The mean nearest-neighbour distance to the power $q$ is the expected value of the average distance to the power $q$ to the nearest neighbour of points of the process (conditional on there being at least two points),

$$
\hat{r}_{\alpha \mu, q}:=\mathrm{E}_{\alpha \mu}^{\geq 2} \sum_{i=1}^{k} \underline{r}\left(x_{i}, N\right)^{q}=\mathrm{E}_{\alpha \mu}^{\geq 2}\left[\frac{1}{\|N\|} \int_{X} \underline{r}\left(x, N-\delta_{x}\right)^{q} N(\mathrm{~d} x)\right] .
$$

Using the same notation as in formula (1.1) this is equal to

$$
\hat{r}_{\alpha \mu}:=\mathrm{E}_{\mu}^{\geq 2}\left[\frac{1}{\|N\|} \sum_{i} \min _{j \neq i} \operatorname{dist}\left(x_{i}, x_{j}\right)^{q}\right] .
$$

Let $\rho_{x}(\beta)$ denote the radius of a ball centered at $x$ and containing the $\beta$-part of the measure $\mu$ (in the left-continuous and right-continuous versions); namely, let us define the functions

$$
\varrho_{x}(\beta):=\sup \left\{r: \mu\left(B_{x}(r)\right)<\beta\right\}=\inf \left\{r: \mu\left(B_{x}(r)\right) \geq \beta\right\}
$$

and

$$
\bar{\rho}_{x}(\beta):=\inf \left\{r: \mu\left(B_{x}(r)\right)>\beta\right\}=\sup \left\{r: \mu\left(B_{x}(r)\right) \leq \beta\right\} .
$$

Let us also introduce the following notation:

$$
\begin{aligned}
\widetilde{r}_{\alpha \mu, q} & :=\int_{X} \hat{r}_{\alpha \mu, q}(x) \mu(\mathrm{d} x), \\
\widetilde{\rho}_{\mu, \beta, q} & :=\int_{X} \bar{\rho}_{x}(\beta)^{q} \mu(\mathrm{d} x) .
\end{aligned}
$$


We will use the following ad hoc notation for dimensions:

$$
\begin{aligned}
& \overline{\operatorname{dim}}_{B P}(\mu, q):=\limsup _{\alpha \rightarrow \infty} \frac{-q \log \alpha}{\log \hat{r}_{\alpha \mu, q}}, \\
& \overline{\operatorname{dim}}_{B P}^{\prime}(\mu, q):=\limsup _{\alpha \rightarrow \infty} \frac{-q \log \alpha}{\log \widetilde{r}_{\alpha \mu, q}}, \\
& \overline{\operatorname{dim}}_{i n v}(\mu, q):=\limsup _{\beta \rightarrow 0} \frac{q \log \beta}{\log \widetilde{\rho}_{\mu, \beta, q}} .
\end{aligned}
$$

$B P$ stands for Badii-Politi, while $i n v$ means inverted and will be commented upon in the next section. $\overline{\operatorname{dim}}_{B P}, \overline{\operatorname{dim}}_{B P}^{\prime}$ and $\overline{\operatorname{dim}}_{i n v}$ are defined analogously with limit inferior. Note that in the definitions of all dimensions we use the conventions $\frac{1}{0}=\infty$ and $\frac{1}{\infty}=0$.

Let us now recall the definition of local dimensions ( $c f$. e.g. [9]):

Definition 1.4. The local lower dimension of the measure $\mu$ at the point $x$ is defined by the formula

$$
\underline{d}_{\mu}(x):=\liminf _{r \rightarrow 0} \frac{\log \mu\left(B_{x}(r)\right)}{\log r} .
$$

The local upper dimension $\bar{d}_{\mu}(x)$ is defined similarly, replacing lim inf by lim sup. Note that nothing changes if we take a closed ball instead of an open ball in the definition.

We will say that a measure $\mu$ has a finite $q$-th moment in $x \in X$ if $M_{x}^{q}(\mu)=$ $\int \operatorname{dist}(x, y)^{q} \mu(\mathrm{d} y)<\infty$. Note that for $q>0$ a measure has a finite $q$-th moment in $x$ if and only if it has a finite $q$-th moment at all points. Now we can state the main results.

Theorem 1.5. If $x \in \operatorname{supp} \mu$ and $\mu(x)$ has a finite $q$-th moment at $x$, then the following equalities hold:

and

$$
\limsup _{\alpha \rightarrow \infty} \frac{-q \log \alpha}{\log \hat{r}_{\alpha \mu, q}(x)}=\bar{d}_{\mu}(x)
$$

$$
\liminf _{\alpha \rightarrow \infty} \frac{-q \log \alpha}{\log \hat{r}_{\alpha \mu, q}(x)}=\underline{d}_{\mu}(x) .
$$

Proposition 1.6. If $X$ is separable and $\mu$ has a finite $q$-th moment for $q>0$ or $\int M_{x}^{q}(\mu) \mu(\mathrm{d} x)<\infty$ for $q<0$, then we have

$$
\overline{\operatorname{dim}}_{B P}(\mu, q)=\overline{\operatorname{dim}}_{B P}(\mu, q)=\overline{\operatorname{dim}}_{\text {inv }}(\mu, q)
$$

and

$$
\underline{\operatorname{dim}}_{B P}(\mu, q)=\underline{\operatorname{dim}}_{B P}^{\prime}(\mu, q)=\underline{\operatorname{dim}}_{i n v}(\mu, q) .
$$

Theorem 1.7. If $q>0$ and $\mu$ has a finite $q$-th moment, then for $\mu$-a.a. $x \in X$ we have $\bar{d}_{\mu}(x) \leq \overline{\operatorname{dim}}_{\text {inv }}(\mu, q)$.

The inequality cannot be replaced by equality:

Example 1.8. Let $a_{n}=1 / 2^{n}$ and $x_{n}=1-\sum_{k=1}^{n-1} a_{n}$ (i.e. $\left.x_{1}=1\right)$ and let us define $\mu^{*}=\sum_{n \in \mathbb{N}} a_{n} \delta_{x_{n}}$. We have $d_{\mu^{*}}\left(x_{k}\right)=0$ for all $k$; therefore $d_{\mu^{*}}(x)=0$ for 
$\mu^{*}$-a.a. $x$. Nevertheless, if $\beta \in\left[a_{n}, a_{n-1}\right)$, then $a_{n} \leq \bar{\rho}_{x_{k}}(\beta) \leq a_{n-1}$ for $k \geq n$ and $\bar{\rho}_{x_{k}}(\beta)=0$ for $k<n$; thereby we have

$$
\underline{\operatorname{dim}}_{i n v}\left(\mu^{*}, q\right)=\overline{\operatorname{dim}}_{i n v}\left(\mu^{*}, q\right)= \begin{cases}0, & q \leq 0, \\ \frac{q}{1+q}, & q>0 .\end{cases}
$$

\section{RELATion to GENERALized Dimension}

It is clear that for some 'regular' fractal measures, the local dimension exists and is equal in a.e. point, and, moreover, most commonly used dimensions are equal. For more complicated or strange, so-called multifractal, measures we need a more subtle characterisation, such as spectral dimension. The most common one is called (in its different versions) generalized dimension, or Rényi or Hentschel-Procaccia dimension spectrum; cf. [12, 18. This spectrum may be defined by a convenient formula:

$$
\overline{\operatorname{dim}}_{R}(\mu, q)=\limsup _{r \rightarrow 0} \frac{\log \int_{X} \mu(B(x, r))^{q-1} \mu(\mathrm{d} x)}{(q-1) \log r},
$$

for $q \neq 1$, which is equivalent to the original definition in most cases (see [10, 17, 18). It seems natural that one could calculate a similar value in the inverted fashion, namely substitute for $r$ the radius of a ball containing a given part of measure $\mu$, namely $\bar{\rho}_{x}$, and taking, analogously, the $\mu$-average of $\bar{\rho}_{x}$. Thus we obtain $\overline{\operatorname{dim}}_{i n v}(\mu, q)$.

Let us notice first the following property of the inverted dimension:

Lemma 2.1. Functions $\underline{\operatorname{dim}}_{i n v}(\mu, q)$ and $\overline{\operatorname{dim}}_{\text {inv }}(\mu, q)$ are nondecreasing in $q$ for $q \in(-\infty, 0)$ and $q \in(0, \infty)$. If $\mu$ has a bounded support, then they are also continuous.

Now, we have the following relation of the inverted dimension and Rényi dimension, which was suggested by Badii and Politi.

Theorem 2.2. If $\mu$ has a compact support, then $\underline{\operatorname{dim}}_{i n v}\left(\mu,(1-q) \underline{\operatorname{dim}}_{R}(\mu, q)\right)=$ $\underline{\operatorname{dim}}_{R}(\mu, q)$ for $q>1$ and $\overline{\operatorname{dim}}_{\text {inv }}\left(\mu,(1-q) \overline{\operatorname{dim}}_{R}(\mu, q)\right)=\overline{\operatorname{dim}}_{R}(\mu, q)$ for $q<1$.

Remark 2.3. Functions $\underline{\operatorname{dim}}_{R}(\mu, q)$ and $\overline{\operatorname{dim}}_{R}(\mu, q)$ are defined here for $q \neq 1$, while $\overline{\operatorname{dim}}_{i n v}(\mu, q)$ and $\underline{\operatorname{dim}}_{i n v}(\mu, q)$ are defined for $q \neq 0$. Therefore Theorem 2.2 and Lemmas 3.6 and 3.7 do not work if the argument of the dimension function is out of the domain.

\section{Proofs}

Let us firstly notice some important facts about nearest-neighbour distances.

Remark 3.1. Note that

$$
\hat{r}_{\alpha \mu, q}(x)=\int_{0}^{\infty} \frac{e^{-\alpha \mu\left(B_{x}\left(r^{1 / q}\right)\right)}-e^{\alpha}}{1-e^{\alpha}} \mathrm{d} r
$$

for $q>0$ and

$$
\hat{r}_{\alpha \mu, q}(x)=\int_{0}^{\infty} \frac{1-e^{-\alpha \mu\left(B_{x}\left(r^{1 / q}\right)\right)}}{1-e^{-\alpha}} \mathrm{d} r
$$

for $q<0$. 
Lemma 3.2. Take arbitrary $x \in \operatorname{supp} \mu$ and $\alpha \geq 1$. The mean closest point $q$ distance $\hat{r}_{\alpha \mu, q}(x)$ at $x$ is finite if and only if $\mu$ has a finite $q$-th moment at $x$.

Proof. Take $q>0$ and notice that

$$
\begin{aligned}
& M_{x}^{q}(\mu)=\int_{0}^{\infty} \mu\left(\left\{y: \operatorname{dist}(x, y)^{q} \geq s\right\}\right) \mathrm{d} s \\
&=\int_{0}^{\infty} \mu\left(X \backslash B_{x}\left(s^{1 / q}\right)\right) \mathrm{d} s=\int_{0}^{\infty}\left[1-\mu\left(B_{x}\left(s^{1 / q}\right)\right)\right] \mathrm{d} s .
\end{aligned}
$$

Using (3.1) and knowing that $\frac{1-t}{e^{\alpha}-1} \leq \frac{e^{-\alpha t}-e^{-\alpha}}{1-e^{-\alpha}} \leq 1-t$, for $t \in[0,1]$, we get $M_{x}^{q}(\mu) /\left(e^{\alpha}-1\right) \leq \hat{r}_{\alpha \mu, q}(x) \leq M_{x}^{q}(\mu)$. For $q<0$ we have $M_{x}^{q}(\mu)=\int_{0}^{\infty} \mu\left(B_{x}\left(s^{1 / q}\right)\right) \mathrm{d} s$. Knowing that $t \leq \frac{1-e^{-\alpha t}}{1-e^{-\alpha}} \leq \frac{\alpha t}{1-e^{-\alpha}}$, for $t \in[0,1]$, we get $M_{x}^{q}(\mu) \leq \hat{r}_{\alpha \mu, q}(x) \leq$ $\frac{\alpha}{1-e^{-\alpha}} M_{x}^{q}(\mu)$.

Remark 3.3. Notice that the functions $\bar{\rho}_{x}$ and $\varrho_{x}$ are nondecreasing and satisfy inter alia the following inequalities:

$$
\begin{gathered}
\bar{\rho}_{x}\left(\mu\left(B_{x}(r)\right)\right) \geq r, \\
\mu\left(B_{\varrho_{x}(\beta)}\right) \leq \beta, \\
\mu\left(\bar{B}_{\varrho_{x}(\beta)}\right) \geq \beta,
\end{gathered}
$$

which we need later.

We use also the following fact:

Remark 3.4. Let $h:[a, b] \rightarrow[h(a), h(b)]$ be a nondecreasing function and let $g:$ $[h(a), h(b)] \rightarrow \mathbb{R}$ be continuously differentiable. Define

$$
\begin{aligned}
& \underline{h^{-1}}(t)=\inf \{s: h(s) \geq t\}, \\
& \overline{h^{-1}}(t)=\sup \{s: h(s) \leq t\} .
\end{aligned}
$$

Then

$$
\begin{aligned}
\int_{a}^{b} g(h(s)) \mathrm{d} s & =b g(h(b))-a g(h(a))-\int_{h(a)}^{h(b)} g^{\prime}(t) \underline{h^{-1}}(t) \mathrm{d} t \\
& =b g(h(b))-a g(h(a))-\int_{h(a)}^{h(b)} g^{\prime}(t) \overline{h^{-1}}(t) \mathrm{d} t .
\end{aligned}
$$

Furthermore, note that if $b=\infty$ and the limit $h(b):=\lim _{t \rightarrow \infty} h(t)$ is finite and $g$ is decreasing and if moreover integrals on both sides are convergent, then the above formula also holds (in such a case we have $b g(h(b))=\lim _{t \rightarrow \infty} t g(h(t))=0$ ).

Proof. Notice that for a differentiable and strictly increasing $h$ that fact is obvious (integration by substitution plus the formula for the derivative of an inverse function and then integration by parts) and of course $\overline{h^{-1}}=\underline{h^{-1}}=h^{-1}$. For an arbitrary nondecreasing function $h:[a, b] \rightarrow[h(a), h(b)]$ the functions under the integrals are both Lebesgue and Riemann integrable, and we can approximate the functions by step functions.

Lemma 3.5. If $q>0$ and $\hat{r}_{\mu, q}(x)$ is finite, then we have

$$
\hat{r}_{\alpha \mu, q}(x) \geq \frac{1}{2 e} \bar{\rho}_{x}(1 / \alpha)^{q}
$$


for all $\alpha>c$ with some $c>1$ and

$$
\hat{r}_{\alpha \mu, q}(x) \leq \varrho_{x}\left(\alpha^{\varepsilon-1}\right)^{q}+\frac{\alpha e^{-\alpha^{\varepsilon}}}{1-e^{-\alpha}} M_{x}^{q}(\mu)
$$

for every $0<\varepsilon<1$ and $\alpha>1$. Moreover, if $q<0$ and $\hat{r}_{\mu, q}(x)$ is finite, then we have

$$
\hat{r}_{\alpha \mu, q}(x) \geq\left(1-e^{-1}\right) \bar{\rho}_{x}(1 / \alpha)^{q}
$$

and

$$
\hat{r}_{\alpha \mu, q}(x) \leq \varrho_{x}\left(\alpha^{\varepsilon-1}\right)^{q}+\frac{\alpha}{1-e^{-\alpha}} M_{x}^{q}(\mu) .
$$

Proof. We use the Remark 3.4 to the formula (3.1) with $h(r)=\mu\left(B_{x}\left(r^{1 / q}\right)\right)$ and $g(s)=\frac{e^{-\alpha s}-e^{\alpha}}{1-e^{\alpha}}$, and we get $\bar{h}^{-1}(\beta)=\bar{\rho}(\beta)^{q}$, so

$$
\hat{r}_{\alpha \mu, q}(x)=\int_{0}^{1} \frac{\alpha e^{-\alpha s}}{1-e^{-\alpha}} \bar{\rho}_{x}(s)^{q} \mathrm{~d} s .
$$

Thanks to Lemma 3.2, the assumptions of Remark 3.4 are satisfied also for unbounded support of $\mu$. Using (3.10), let us calculate

$$
\begin{aligned}
\hat{r}_{\alpha \mu, q}(x) & =\int_{0}^{1 / \alpha} \frac{\alpha e^{-\alpha s}}{1-e^{-\alpha}} \bar{\rho}_{x}(s)^{q} \mathrm{~d} s+\int_{1 / \alpha}^{1} \frac{\alpha e^{-\alpha s}}{1-e^{-\alpha}} \bar{\rho}_{x}(s)^{q} \mathrm{~d} s \\
& \geq \bar{\rho}_{x}(1 / \alpha)^{q} \int_{1 / \alpha}^{1} \frac{\alpha e^{-\alpha s}}{1-e^{-\alpha}} \mathrm{d} s=\bar{\rho}_{x}(1 / \alpha)^{q} e^{-} \frac{1-e^{-(\alpha-1)}}{1-e^{-\alpha}},
\end{aligned}
$$

which gives the first inequality. Similarly we have

$$
\begin{aligned}
\hat{r}_{\alpha \mu}(x) & =\int_{0}^{\alpha^{\varepsilon-1}} \frac{\alpha e^{-\alpha s}}{1-e^{-\alpha}} \varrho_{x}(s)^{q} \mathrm{~d} s+\int_{\alpha^{\varepsilon-1}}^{1} \frac{\alpha e^{-\alpha s}}{1-e^{-\alpha}} \varrho_{x}(s)^{q} \mathrm{~d} s \\
& \leq \varrho_{x}\left(\alpha^{\varepsilon-1}\right)^{q} \int_{0}^{\alpha^{\varepsilon-1}} \frac{\alpha e^{-\alpha s}}{1-e^{-\alpha}} \mathrm{d} s+\frac{\alpha e^{-\alpha^{\varepsilon}}}{1-e^{-\alpha}} \int_{\alpha^{\varepsilon-1}}^{1} \varrho_{x}(s)^{q} \mathrm{~d} s,
\end{aligned}
$$

which completes the proof of the second inequality, because the first integral is less than one and $\int_{0}^{1} \varrho_{x}(s)^{q} \mathrm{~d} s=M_{x}^{q}(\mu)$ by Remark 3.4. A proof of the last two inequalities is analogous.

Proof of Theorem 1.5. First, note that $\mu\left(B_{x}\left(\varrho_{x}(1 / \alpha)\right)\right) \leq \frac{1}{\alpha} \leq \mu\left(\bar{B}_{x}\left(\varrho_{x}(1 / \alpha)\right)\right)$, so

$$
\frac{-\log \mu\left(B_{x}\left(\varrho_{x}(1 / \alpha)\right)\right)}{\log \varrho_{x}(1 / \alpha)} \geq \frac{-\log \alpha}{\log \varrho_{x}(1 / \alpha)} \geq \frac{-\log \mu\left(\bar{B}_{x}\left(\varrho_{x}(1 / \alpha)\right)\right)}{\log \varrho_{x}(1 / \alpha)}
$$

and thereby $\underline{d}_{\mu}(x)$ and $\bar{d}_{\mu}(x)$ are equal to the lower and upper limit of $\frac{-\log \alpha}{\log \underline{\varrho}_{x}(1 / \alpha)}$, respectively.

Now notice that if $x$ is an atom of $\mu($ i.e. $\mu(\{x\})>0)$, then both $\bar{d}_{\mu}(x)=\underline{d}_{\mu}(x)=$ 0 and $\lim _{\alpha \rightarrow \infty} \frac{-\log \alpha}{\log \hat{r}_{\alpha \mu, q}(x)}=0$ thanks to inequality (3.7) and the fact that $\varrho_{x}(\beta)=0$ for small $\beta$. Therefore, we can assume that $\lim _{r \rightarrow 0} \mu\left(B_{x}(r)\right)=0$. Note that for a positive $q$, the value of $\hat{r}_{\alpha \mu, q}(x)$ tends to 0 as $\alpha$ goes to $\infty($ since $x \in \operatorname{supp} \mu$ ), while for a negative $q$ it tends to infinity.

Using the inequalities (3.6) and (3.8) from Lemma 3.5 we get

$$
\frac{-q \log \alpha}{\log \hat{r}_{\alpha \mu, q}(x)} \geq \frac{-q \log \alpha}{q \log \varrho_{x}(1 / \alpha)-\log 2 e},
$$


and thereby both

$$
\limsup _{\alpha \rightarrow \infty} \frac{-\log \alpha}{\log \hat{r}_{\alpha \mu}(x)} \geq \bar{d}_{\mu}(x)
$$

and

$$
\liminf _{\alpha \rightarrow \infty} \frac{-\log \alpha}{\log \hat{r}_{\alpha \mu}(x)} \geq \bar{d}_{\mu}(x) .
$$

To prove the inverse inequalities let us assume that

$$
\limsup _{r \rightarrow 0} \frac{-q \log \alpha}{q \log \varrho_{x}(1 / \alpha)}<d_{1}<d_{0}<d
$$

for some $d, d_{0}, d_{1}>0$. In the case when $q>0$ there exists an $s_{0}>0$ such that $\varrho_{x}(s) \leq s^{1 / d_{1}}$ for all $s<s_{0}$. Take an $\varepsilon>0$ such that $\frac{d_{1}}{1-\varepsilon} \leq d_{0}$. Thus, using inequality (3.7) we get

$$
\hat{r}_{\alpha \mu, q}(x) \leq \alpha^{-\frac{q(1-\varepsilon)}{d_{1}}}+\frac{\alpha e^{-\alpha^{\varepsilon}}}{1-e^{-\alpha\|\mu\|}} M_{x}^{q}(\mu)
$$

if $\alpha^{\varepsilon-1}<s_{0}$. Since $2 \alpha^{1+q / d_{0}} e^{-\alpha^{\varepsilon}}$ converges to 0 as $\alpha \rightarrow \infty$ the second summand on the right hand side of (3.13) is less than $\alpha^{-\frac{q}{d_{0}}}$ for large $\alpha$; thereby we have

$$
\hat{r}_{\alpha \mu}(x) \leq 2 \alpha^{-\frac{q}{d_{0}}},
$$

which for large $\alpha$ is less than $\alpha^{-q / d}$. Thus

$$
\frac{-q \log \alpha}{\log \hat{r}_{\alpha \mu, q}(x)} \leq d
$$

for any $d>\bar{d}_{\mu}(x)$ and all sufficiently large $\alpha$, so the inequality inverse to (3.11) holds. A similar reasoning proves the last inequality when $q>0$, and analogous calculations may be done for $q<0$ with the use of inequality (3.9).

Proof of Proposition 1.6. First, let us prove that $\overline{\operatorname{dim}}_{B P}(\mu, q)=\overline{\operatorname{dim}}_{B P}^{\prime}(\mu, q)$ and $\underline{\operatorname{dim}}_{B P}(\mu, q)=\underline{\operatorname{dim}}_{B P}^{\prime}(\mu, q)$. The distance to the closest point function $\underline{r}(x, N)^{q}$ may be approximated by finite sums of functions of the form $\chi_{B, A}(x, N)=$ $\mathbb{1}_{B}(x) \mathbb{1}_{\{\nu \in \mathcal{N}: \nu(A)=0\}}(N)$, where $A, B \in \mathcal{B}(X)$ and $B \subset A$. Precisely, let $\left\{x_{i}\right\}_{i \in \mathbb{N}}$ be a dense subset of $X$ and for fixed $n \in \mathbb{N}$ let $\left\{B_{i}^{(n)}\right\}_{i \in N}$ be a family of pairwise disjoint sets such that $B_{i}^{(n)} \subset B_{x_{i}}\left(\frac{1}{2^{n}}\right)$. Now define

$$
f_{n}(x, N)=\sum_{i=1}^{\infty} \mathbb{1}_{B_{i}^{(n)}}(x) \sum_{k=2}^{2^{2 n}} \frac{(k-1)^{q}-(k-2)^{q}}{2^{q n}} \mathbb{1}_{\left\{\nu \in \mathcal{N}: \nu\left(B_{x_{i}}\left(k / 2^{n}\right)\right)=0\right\}}(N),
$$

and notice that $f_{n}(x, N) \leq f_{n+1}(x, N) \leq \underline{r}(x, N)^{q}$ for every $n \in \mathbb{N}, x \in X$ and $N \in \mathcal{N}$ and moreover $f_{n}$ converges pointwise to $\underline{r}^{q}$ as $n$ goes to infinity. Note that for $q<0$ we need to use a different approximation, although we omit this case here. Take $B \subset A \subset X$ and let us now calculate

$$
\begin{aligned}
& \text { 14) } \quad \mathrm{E}_{\alpha \mu}^{\geq 2}\left[\frac{1}{\|N\|} \int_{X} \chi\left(x, N-\delta_{x}\right) N(\mathrm{~d} x)\right]= \\
& \mathrm{E}_{\alpha \mu}^{\geq 2}\left[\frac{1}{\|N\|} \sum_{i=1}^{\|N\|} \mathbb{1}_{B}\left(x_{i}\right) \mathbb{1}_{\{\nu \in \mathcal{N}: \nu(A)=0\}}\left(N-\delta_{x}\right)\right]=\alpha \mu(B) \frac{\sum_{n=2}^{\infty} \frac{\alpha^{n-1} \mu(X \backslash A)^{n-1}}{n !}}{\sum_{n=2}^{\infty} \frac{\alpha^{n}}{n !}}
\end{aligned}
$$


and

$$
\begin{aligned}
\int_{X} \mathrm{E}_{\alpha \mu}^{\geq 2} \chi(x, N) \mu(\mathrm{d} x) \\
\quad=\int_{X}\left[\mathbb{1}_{B}(x) \frac{\operatorname{Prob}_{\alpha \mu}(N(A)=0 \text { and } N(X) \geq 2)}{\operatorname{Prob}_{\alpha \mu}(N(X) \geq 2)}\right] \mu(\mathrm{d} x) \\
\quad=\frac{\mu(B) \sum_{n=2}^{\infty} \frac{\alpha^{n} \mu(X \backslash A)^{n}}{n !}}{\sum_{n=2}^{\infty} \frac{\alpha^{n}}{n !}}=\mu(X \backslash A) \alpha \mu(B) \frac{\sum_{n=2}^{\infty} \frac{\alpha^{n-1} \mu(X \backslash A)^{n-1}}{n !}}{\sum_{n=2}^{\infty} \frac{\alpha^{n}}{n !}} .
\end{aligned}
$$

Therefore, (3.15) is less than (3.14). Since sums of expressions of the form (3.14) approximate $\hat{r}_{\alpha \mu, q}$, while sums of (3.15) approximate $\int_{X} \mathrm{E}^{\geq 2} \underline{r}(x, N)^{q} \mu(\mathrm{d} x)$, which is equal to $\widetilde{r}_{\alpha \mu, q}$ up to an exponentially decreasing term in the limit of $\alpha \rightarrow \infty$, we have proved that $\lim \sup _{\alpha \rightarrow \infty} \frac{-q \log \alpha}{\log \hat{r}_{\alpha \mu, q}} \geq \lim \sup _{\alpha \rightarrow \infty} \frac{-q \log \alpha}{\log \tilde{r}_{\alpha \mu, q}}$ and $\liminf \inf _{\alpha \rightarrow \infty} \frac{-q \log \alpha}{\log \hat{r}_{\alpha \mu, q}} \geq$ $\lim \inf _{\alpha \rightarrow \infty} \frac{-q \log \alpha}{\log \tilde{r}_{\alpha \mu, q}}$. To obtain the reverse inequalities notice that, similarly to (3.14) and (3.15), we have

$$
\begin{aligned}
& \frac{1}{\alpha} \mathrm{E}_{\alpha \mu}^{\geq 2}\left[\int_{X} \chi\left(x, N-\delta_{x}\right) N(\mathrm{~d} x)\right] \\
& \quad=\frac{\alpha \mu(B) \sum_{n=1}^{\infty} \frac{\alpha^{n} \mu(X \backslash A)^{n}}{n !}}{\alpha \sum_{n=2}^{\infty} \frac{\alpha^{n}}{n !}}=\frac{\operatorname{Prob}_{\alpha \mu}(\|N\| \geq 1)}{\operatorname{Prob}_{\alpha \mu}(\|N\| \geq 2)} \int_{X} \mathrm{E}_{\alpha \mu}^{\geq 1} \chi(x, N) \mu(\mathrm{d} x)
\end{aligned}
$$

and thereby $\hat{r}_{\alpha \mu, q}^{\prime}=\frac{1}{\alpha} \mathrm{E}_{\alpha \mu}^{\geq 2}\left[\int_{X} \underline{r}\left(x, N-\delta_{x}\right)^{q} N(\mathrm{~d} x)\right]$ is asymptotically equal to $\widetilde{r}_{\alpha \mu, q}$. Now we need to prove that

$$
\limsup _{\alpha \rightarrow \infty} \frac{-q \log \alpha}{\log \hat{r}_{\alpha \mu, q}} \leq \limsup _{\alpha \rightarrow \infty} \frac{-q \log \alpha}{\log \hat{r}_{\alpha \mu, q}^{\prime}} .
$$

To that end let $\xi_{N}$ denote the random variable $\frac{1}{\|N\|} \int_{X} \underline{r}\left(x, N-\delta_{x}\right)^{q} N(\mathrm{~d} x)$ and set $p_{\alpha, n}=\operatorname{Prob}_{\alpha \mu}(\|N\|=n)=\frac{\alpha^{n}}{n !} e^{-\alpha}$. Note that

$$
\begin{aligned}
& \frac{1}{\alpha} \mathrm{E}_{\alpha \mu}^{\geq 2}\left[\int_{X} \underline{r}\left(x, N-\delta_{x}\right)^{q} N(\mathrm{~d} x)\right]=\frac{1}{\alpha} \mathrm{E}_{\alpha \mu}^{\geq 2}\left[\|N\| \xi_{N}\right] \\
& \geq \frac{1}{\alpha} \sum_{n=\left\lfloor\frac{\alpha}{2}\right\rfloor+1}^{\infty} p_{\alpha, n} n \mathrm{E}_{\alpha \mu}^{\geq 2}\left[\xi_{N} \mid\|N\|=n\right] \geq \sum_{n=\left\lfloor\frac{\alpha}{2}\right\rfloor+1}^{\infty} p_{\alpha, n} \mathrm{E}_{\alpha \mu}^{\geq 2}\left[\xi_{N} \mid\|N\|=n\right] \\
& =\hat{r}_{\alpha \mu, q}-\sum_{n=2}^{\left\lfloor\frac{\alpha}{2}\right\rfloor} p_{\alpha, n} \mathrm{E}_{\alpha \mu}^{\geq 2}\left[\xi_{N} \mid\|N\|=n\right] \geq \hat{r}_{\alpha \mu, q}-\mathrm{E}_{\alpha \mu}^{\geq 2}\left[\xi_{N} \mid\|N\|=2\right] \sum_{n=2}^{\left\lfloor\frac{\alpha}{2}\right\rfloor} \frac{\alpha^{n}}{n !} e^{-\alpha},
\end{aligned}
$$

where $\lfloor a\rfloor$ denotes the largest integer not greater than $a$. Now it suffices to estimate that the last summand does not change the value of the limit in (3.16). We have

$$
\sum_{n=2}^{\left\lfloor\frac{\alpha}{2}\right\rfloor} \frac{\alpha^{n}}{n !} e^{-\alpha} \leq e^{-\alpha} \sum_{n=2}^{\left\lfloor\frac{\alpha}{2}\right\rfloor} \frac{\alpha^{n}}{n !} \leq e^{-\alpha}\left\lfloor\frac{\alpha}{2}\right\rfloor \frac{(\alpha \mu)^{\left\lfloor\frac{\alpha}{2}\right\rfloor}}{\left\lfloor\frac{\alpha}{2}\right\rfloor !}=e^{-\alpha} \frac{(\alpha \mu)^{\left\lfloor\frac{\alpha}{2}\right\rfloor}}{\Gamma\left(\left\lfloor\frac{\alpha}{2}\right\rfloor\right)},
$$

which tends exponentially to zero. Therefore, we have $\lim \sup _{\alpha \rightarrow \infty} \frac{-q \log \alpha}{\log \hat{r}_{\alpha \mu, q}} \leq$ $\limsup \operatorname{su}_{\alpha \rightarrow \infty} \frac{-q \log \alpha}{\log \tilde{r}_{\alpha \mu, q}}$ and $\liminf \inf _{\alpha \rightarrow \infty} \frac{-q \log \alpha}{\log \hat{r}_{\alpha \mu, q}} \leq \liminf \inf _{\alpha \rightarrow \infty} \frac{-q \log \alpha}{\log \tilde{r}_{\alpha \mu, q}}$. 
Now, let us prove the second part, namely $\overline{\operatorname{dim}}_{B P}^{\prime}(\mu, q)=\overline{\operatorname{dim}}_{i n v}(\mu, q)$ and $\underline{\operatorname{dim}}_{B P}^{\prime}(\mu, q)=\underline{\operatorname{dim}}_{i n v}(\mu, q)$. By integrating the inequality (3.6) or (3.8) with respect to $\mu$ we get

$$
\hat{r}_{\alpha \mu, q}^{\prime} \geq 2 e \int_{X} \bar{\rho}_{x}(1 / \alpha)^{q} \mu(\mathrm{d} x)
$$

which immediately gives $\overline{\operatorname{dim}}_{B P}^{\prime} \mu \geq \overline{\operatorname{dim}}_{\text {inv }} \mu$ and $\underline{\operatorname{dim}}_{B P}^{\prime} \mu \geq \underline{\operatorname{dim}}_{i n v} \mu$.

To obtain the reverse inequalities we use the inequality (3.7) and get

$$
\widetilde{r}_{\alpha \mu, q} \leq \widetilde{\rho}_{\mu, \alpha^{\varepsilon-1}, q}+\frac{\alpha e^{-\alpha^{\varepsilon}}}{1-e^{-\alpha}} \int_{X} M_{x}^{q}(\mu) \mu(\mathrm{d} x) .
$$

Note, however, that the last integral is finite for positive $q$ if $\mu$ has a finite $q$-th moment. The rest of the proof is similar to that of Theorem 1.5. This time we write the proof of the liminf part. Let $d=\liminf _{\beta \rightarrow 0} \frac{q \log \beta}{\log \widetilde{\rho}_{\mu, \beta, q}}$ and take arbitrary $\varepsilon>0$. There exist a sequence $\left\{\beta_{n}\right\}_{n \in N}$ decreasing to 0 such that $\beta_{n}^{\frac{q}{d+\varepsilon}}>\int \rho_{x}\left(\beta_{n}\right)^{q} \mu(\mathrm{d} x)$. Let $\alpha_{n}=\beta_{n}^{\frac{1}{1-\varepsilon}}$. Then $\alpha_{n} \rightarrow 0$ and

$$
\widetilde{r}_{\alpha_{n} \mu, q} \leq \alpha_{n}^{q \frac{1-\varepsilon}{d+\varepsilon}}+\frac{\alpha e^{-\alpha^{\varepsilon}}}{1-e^{-\alpha}} M_{x_{0}}^{q}(\mu) .
$$

Since the latter summand is for large $\alpha$ less than the former one, we have $\widetilde{r}_{\alpha_{n} \mu, q} \leq$ $2 \alpha_{n}^{q \frac{1-\varepsilon}{d+\varepsilon}}$, which is less than $\alpha_{n}^{\varepsilon} \alpha_{n}^{q \frac{1-\varepsilon}{d+\varepsilon}}$ for large $n$. Therefore $\underline{\operatorname{dim}}_{B P}^{\prime} \mu \leq \frac{d+\varepsilon}{1-\varepsilon-(d+\varepsilon) \varepsilon}$ for arbitrarily small $\varepsilon$. A proof for negative $q$ is analogous, provided we have the additional assumption that $\int M_{x}^{q}(\mu) \mu(\mathrm{d} x)<\infty$.

Proof of Theorem [1.7. We use a similar method as in [19. Take $d, d_{0}$ and $d_{1}$ such that

$$
d>d_{0}>d_{1}>\limsup _{\beta \downarrow 0} \frac{q \log \beta}{\log \int_{X} \bar{\rho}_{x}(\beta)^{q} \mu(\mathrm{d} x)}
$$

and let $A(\beta)=\left\{x \in X: \bar{\rho}_{x}(\beta)>\beta^{1 / d_{0}}\right\}$. Note that for sufficiently small $\beta$ we have

$$
\int_{X} \bar{\rho}_{x}(\beta)^{q} \mu(\mathrm{d} x)<\beta^{q / d_{1}}
$$

and thereby $\mu(A(\beta))<\beta^{\frac{q}{d_{1}}-\frac{q}{d_{0}}}$. Define a sequence $\beta_{m}=2^{-\left(d / d_{0}\right)^{m}}$ and observe that, since $\sum_{m=1}^{\infty} \beta_{m}^{\frac{q}{d_{1}}-\frac{q}{d_{0}}}<\infty$, by the Borel-Cantelli lemma we have

$$
\mu\left(\bigcap_{n=1}^{\infty} \bigcup_{m=n}^{\infty} A\left(\beta_{m}\right)\right)=0
$$

Notice, however, that $\left\{x \in X: \bar{d}_{\mu}(x)>d\right\} \subset \bigcap_{n=1}^{\infty} \bigcup_{m=n}^{\infty} A\left(\beta_{m}\right)$. Indeed, if we fix $x \in X$ such that $\bar{d}_{\mu}(x)>d$, then there exists a sequence $\left(r_{n}\right)_{n \in \mathbb{N}}$ convergent to 0 and such that $1-\mu\left(B_{x}\left(r_{n}\right)\right)<r_{n}^{d}$. If we set $s_{n}=r_{n}^{d}$, then $\bar{\rho}_{x}\left(s_{n}\right) \geq \bar{\rho}_{x}\left(\frac{1}{\mu} \mu\left(B_{x}\left(r_{n}\right)\right)\right) \geq$ $s_{n}^{1 / d}$ and moreover $\bar{\rho}_{x}(s)>s^{1 / d_{0}}$ for every $s \in\left[s_{n}, s_{n}^{d_{0} / d}\right]$. Thereby, since for every $n$ we have a $\beta_{m_{n}} \in\left[s_{n}, s_{n}^{d_{0} / d}\right]$, it follows that $x \in A\left(\beta_{m_{n}}\right)$ for every $n$. 
Proof of Lemma 2.1. By Jensen's inequality, if $a>b>0$, then $\int_{X} \bar{\rho}_{x}(\beta)^{a} \mu(\mathrm{d} x)$ $\geq\left[\int_{X} \bar{\rho}_{x}(\beta)^{b} \mu(\mathrm{d} x)\right]^{a / b}$, whilst for $0>a>b$ we have $\int_{X} \bar{\rho}_{x}(\beta)^{a} \mu(\mathrm{d} x)$ $\leq\left[\int_{X} \bar{\rho}_{x}(\beta)^{b} \mu(\mathrm{d} x)\right]^{a / b}$. Thus for both positive and negative $a>b$ we have

$$
\frac{a \log \beta}{\log \int_{X} \bar{\rho}_{x}(\beta)^{a} \mu(\mathrm{d} x)} \geq \frac{b \log \beta}{\log \int_{X} \bar{\rho}_{x}(\beta)^{b} \mu(\mathrm{d} x)},
$$

which proves monotonicity. Moreover, if $a>b>0$, then

$$
\int_{X} \bar{\rho}_{x}(\beta)^{a} \mu(\mathrm{d} x) \leq(\operatorname{diam} \operatorname{supp} \mu)^{\frac{a}{b}-1} \int_{X} \bar{\rho}_{x}(\beta)^{b} \mu(\mathrm{d} x)
$$

so

$$
\frac{a \log \beta}{\log \int_{X} \bar{\rho}_{x}(\beta)^{a} \mu(\mathrm{d} x)} \leq \frac{a}{b} \frac{b \log \beta}{\log (\operatorname{diam} \operatorname{supp} \mu)^{\frac{a}{b}-1} \int_{X} \bar{\rho}_{x}(\beta)^{b} \mu(\mathrm{d} x)},
$$

which assures continuity for positive $q$.

To prove that the dimension functions are continuous for negative $q$, let us notice that for fixed $\beta>0$,

(1) either there exists an $x \in \operatorname{supp} \mu$ such that $\bar{\rho}_{x}(\beta)=0$, which is equivalent to $\mu(\{x\}) \geq \beta$,

(2) or there exists a number $r_{0}>0$ such that $\bar{\rho}_{x}(\beta)>r_{0}$ for all $x \in \operatorname{supp} \mu$. Indeed, if $\bar{\rho}_{x}(\beta)$ were positive for all $x$ but $\inf _{x} \bar{\rho}_{x}(\beta)=0$, then one can check that there exists a sequence $\left(x_{n}\right)_{n \in \mathbb{N}}$ such that $\bar{\rho}_{x_{n}}(\beta) \rightarrow 0$ and $x_{n}$ is convergent to some $x^{*}$. Then $\mu\left(\left\{x^{*}\right\}\right) \geq \beta$.

In case (1) we have $\int \bar{\rho}_{x}(\beta)^{q} \mu(\mathrm{d} x)=\infty$ and $\overline{\operatorname{dim}}_{\text {inv }}(\mu, q)=\underline{\operatorname{dim}}_{\text {inv }}(\mu, q)=0$ for all $q<0$. In case (2), notice first that for fixed $\beta>0$ there exists a point $x_{\beta} \in \operatorname{supp} \mu$ such that $\bar{\rho}_{x_{\beta}}(\beta)<2 \inf _{x \in \operatorname{supp} \mu} \bar{\rho}_{x}(\beta)$. Therefore, $\bar{\rho}_{x}(\beta) \leq 4 \inf _{x \in \operatorname{supp} \mu} \bar{\rho}_{x}(\beta)$ for $x \in \bar{B}\left(x_{\beta}, \bar{\rho}_{x_{\beta}}(\beta)\right)$ and $\mu\left(\bar{B}\left(x_{\beta}, \bar{\rho}_{x_{\beta}}(\beta)\right)\right) \geq \beta$. So $\int_{X} \bar{\rho}_{x}(\beta)^{a} \mu(\mathrm{d} x) \geq$ $4^{a}\left(\inf _{x \in \operatorname{supp} \mu} \bar{\rho}_{x}(\beta)\right)^{a} \beta$ for $a<0$ and thereby $\inf _{x \in \operatorname{supp} \mu} \bar{\rho}_{x}(\beta) \geq \frac{\left(\int \bar{\rho}_{x}(\beta)^{a} \mu(\mathrm{d} x)\right)^{1 / a}}{4 \beta^{1 / a}}$. Then for $b<a<0$,

$$
\begin{array}{r}
\frac{b \log \beta}{\log \int_{X} \bar{\rho}_{x}(\beta)^{b} \mu(\mathrm{d} x)} \geq \frac{b}{a} \frac{a \log \beta}{\log \int_{X} \bar{\rho}_{x}(\beta)^{a} \mu(\mathrm{d} x)+(b-a) \log \frac{\left(\int \bar{\rho}_{x}(\beta)^{a} \mu(\mathrm{d} x)\right)^{1 / a}}{4 \beta^{1 / a}}} \\
=\frac{a \log \beta}{\log \int \bar{\rho}_{x}(\beta)^{a} \mu(\mathrm{d} x)+\frac{a-b}{b} \log 4^{q} \beta},
\end{array}
$$

and continuity of both $\overline{\operatorname{dim}}_{i n v}(\mu, q)$ and $\underline{\operatorname{dim}}_{i n v}(\mu, q)$ follows.

Lemma 3.6. If $\mu$ has a compact support, then $\underline{\operatorname{dim}}_{\text {inv }}\left(\mu,(1-q) \underline{\operatorname{dim}}_{R}(\mu, q)\right) \geq$ $\underline{\operatorname{dim}}_{R}(\mu, q)$ for $q>1$ and $\overline{\operatorname{dim}}_{\text {inv }}\left(\mu,(1-q) \overline{\operatorname{dim}}_{R}(\mu, q)\right) \leq \overline{\operatorname{dim}}_{R}(\mu, q)$ for $q<1$.

Proof. By compactness, for every $r>0$ there exists $\beta>0$ such that $\bar{\rho}_{x}(\beta)<r$ for all $x \in \operatorname{supp} \mu$. Fix small $\varepsilon>0$ and set $\gamma=1-\varepsilon$. There exists $r_{0}>0$ such that

$$
\frac{\log \int \mu(\bar{B}(x, r))^{q-1} \mu(\mathrm{d} x)}{(q-1) \log r}>\underline{\operatorname{dim}}_{R}(\mu, q)-\varepsilon
$$

for $r<r_{0}$ and thereby

$$
r^{\gamma(1-q)\left(\underline{\operatorname{dim}}_{R}(\mu, q)-\varepsilon\right)}<\int \mu(\bar{B}(x, r))^{q-1} \mu(\mathrm{d} x)^{-\gamma}
$$


for $q>1$. Note, moreover, that

$$
\begin{array}{r}
\int \mu\left(\bar{B}\left(y, \rho_{x}(\beta)\right)\right)^{q-1} \mu(\mathrm{d} y) \geq \int_{0}^{1} \mu\left(\left\{y \in X: \rho_{x}(\beta)>\rho_{y}\left(\alpha^{\frac{1}{q-1}}\right)\right\}\right) \mathrm{d} \alpha \\
\geq \beta^{q-1} \mu\left(\left\{y \in X: \rho_{x}(\beta)>\rho_{y}(\beta)\right\}\right)
\end{array}
$$

and

$$
\begin{aligned}
& \int\left[\mu\left(\left\{y \in X: \rho_{x}(\beta)>\rho_{y}(\beta)\right\}\right)\right]^{-\gamma} \mu(\mathrm{d} x) \\
& \quad=\int_{0}^{\infty} \mu\left\{x \in X: \mu\left(\left\{x \in X: \rho_{x}(\beta)>\rho_{y}(\beta)\right\}\right) \leq s^{-1 / \gamma}\right\} \mathrm{d} s \\
& \quad=\int_{0}^{\infty} \min \left\{1, s^{-1 / \gamma}\right\} \mathrm{d} s .
\end{aligned}
$$

Therefore, using (3.17)-(3.19), we get

$$
\int \rho_{x}(\beta)^{\gamma(1-q)\left(\underline{\operatorname{dim}}_{R}(\mu, q)-\varepsilon\right)} \mu(\mathrm{d} x)<\beta^{\gamma(1-q)} \int_{0}^{\infty} \min \left\{1, s^{-1 / \gamma}\right\} \mathrm{d} s .
$$

Thus

$$
\begin{aligned}
& \liminf _{\beta \rightarrow 0} \frac{\gamma(1-q)\left(\underline{\operatorname{dim}}_{R}(\mu, q)-\varepsilon\right) \log \beta}{\log \int \bar{\rho}_{x}(\beta)^{\gamma(1-q)\left(\underline{\operatorname{dim}}_{R}(\mu, q)-\varepsilon\right)} \mu(\mathrm{d} x)} \\
& \quad \geq\left(\underline{\operatorname{dim}}_{R}(\mu, q)-\varepsilon\right) \liminf _{\beta \rightarrow 0} \frac{\gamma(1-q) \log \beta}{\log \beta^{\gamma(1-q)}\left(1+\frac{\gamma}{1-\gamma}\right)}
\end{aligned}
$$

and, by the continuity of $\overline{\operatorname{dim}}_{i n v}(\mu, \cdot)$, the assertion of the theorem for $q>1$ follows. A similar calculation for $q<1$ yields $\overline{\operatorname{dim}}_{i n v}\left(\mu, \gamma(1-q)\left(\overline{\operatorname{dim}}_{R}(\mu, q)+\varepsilon\right)\right) \leq$ $\left(\overline{\operatorname{dim}}_{R}(\mu, q)+\varepsilon\right)$.

Lemma 3.7. If $\mu$ has a compact support, then $\underline{\operatorname{dim}}_{R}\left(\mu, 1-\frac{q}{\underline{\operatorname{dim}}_{i n v}(\mu, q)}\right) \geq \underline{\operatorname{dim}}_{i n v}(\mu, q)$ for $q<0$ and $\overline{\operatorname{dim}}_{R}\left(\mu, 1-\frac{q}{\overline{\operatorname{dim}}_{i n v}(\mu, q)}\right) \leq \overline{\operatorname{dim}}_{i n v}(\mu, q)$ for $q>0$.

Proof. If $\mu$ has no atoms, then the proof is very similar to the previous one. If, however, there are some points $x_{i}$ such that $\mu\left(\left\{x_{i}\right\}\right)>0$, then:

(1) For $q<0$ we have $\underline{\operatorname{dim}}_{i n v}(\mu, q)=0$.

(2) For $q>0$, let $\beta_{0}>0$ be such that $\beta^{-\frac{\gamma q}{\operatorname{dim}_{i n v}(\mu, q)+\varepsilon}}<\int \rho_{y}(\beta)^{q} \mu(\mathrm{d} x)$ if $\beta<\beta_{0}$. We have at most a finite number of points, say $x_{1}, \ldots, x_{n}$, such that $\mu\left(\left\{x_{i}\right\}\right)>\beta_{0}$. Thus, we have

$$
\begin{aligned}
& \int_{X} \mu\left(B_{x}(x, r)\right)^{-\overline{\operatorname{dim}}_{i n v}(\mu, q)+\varepsilon} \mu(\mathrm{d} x) \\
& \leq \int_{X}\left[\int \rho_{y} \mu\left(B_{x}(r)\right)^{q} \mu(\mathrm{d} x),\right]^{-\gamma} \mu(\mathrm{d} x)+\sum_{i=1}^{n} \mu\left(\left\{x_{i}\right\}\right)^{-\frac{\gamma q}{\overline{\operatorname{dim}}_{i n v}(\mu, q)+\varepsilon}},
\end{aligned}
$$


where the last sum (let us denote it by $C_{\varepsilon}$ ) does not depend on $r$. Therefore, following the previous proof, we have

$$
\begin{aligned}
& \left(\overline{\operatorname{dim}}_{i n v}(\mu, q)+\varepsilon\right) \limsup _{r \rightarrow 0} \frac{\log \int_{X} \mu\left(B_{x}(x, r)\right)^{-\frac{\gamma q}{\operatorname{dim}_{i n v}(\mu, q)+\varepsilon}} \mu(\mathrm{d} x)}{-\gamma q \log r} \\
& \leq\left(\overline{\operatorname{dim}}_{i n v}(\mu, q)+\varepsilon\right) \limsup _{r \rightarrow 0} \frac{\log \int_{X}\left[\int \rho_{y} \mu\left(B_{x}(r)\right)^{q} \mu(\mathrm{d} x),\right]^{-\gamma} \mu(\mathrm{d} x)+C_{\varepsilon}}{-\gamma q \log r} \\
& =\left(\overline{\operatorname{dim}}_{i n v}(\mu, q)+\varepsilon\right) \limsup _{r \rightarrow 0} \frac{\log r^{\gamma(1-q)}\left(1+\frac{\gamma}{1-\gamma}\right)}{-\gamma q \log r},
\end{aligned}
$$

which completes the proof of the lemma.

Proof of Theorem 2.2. The proof uses Lemmas 3.6 and 3.7. First, take $\bar{q}>0$ and set $q=1-\frac{\bar{q}}{\underline{\operatorname{dim}}_{i n v}(\mu, \bar{q})}$. Now we have

$$
\underline{\operatorname{dim}}_{R}\left(\mu, 1-\frac{\bar{q}}{\underline{\operatorname{dim}}_{i n v}(\mu, \bar{q})}\right)=\underline{\operatorname{dim}}_{i n v}(\mu, q) \leq \underline{\operatorname{dim}}_{i n v}\left(\mu,(1-q) \underline{\operatorname{dim}}_{R}(\mu, q)\right) .
$$

But $\underline{\operatorname{dim}}_{R}(\mu, q) \geq \underline{\operatorname{dim}}_{i n v}(\mu, \bar{q})$, so $(1-q) \underline{\operatorname{dim}}_{R}(\mu, q) \leq \bar{q}$ and, since $\underline{\operatorname{dim}}_{i n v}(\mu, \cdot)$ is nondecreasing, we have $\underline{\operatorname{dim}}_{i n v}\left(\mu,(1-q) \underline{\operatorname{dim}}_{R}(\mu, q)\right) \leq \underline{\operatorname{dim}}_{i n v}(\mu, \bar{q})$. Hence $\underline{\operatorname{dim}}_{R}\left(\mu, 1-\frac{\bar{q}}{\underline{\operatorname{dim}}_{i n v}(\mu, \bar{q})}\right)=\underline{\operatorname{dim}}_{i n v}(\mu, \bar{q})$. From continuity and monotonicity of $\underline{\operatorname{dim}}_{i n v}(\mu, \cdot)$ it follows that for all $q>1$ there exists a negative $\bar{q}$ such that $q=$ $1-\frac{\bar{q}}{\underline{\operatorname{dim}}_{\text {inv }}(\mu, \bar{q})}$, which proves the former statement of the theorem. The latter one follows analogously.

\section{Concluding Remarks}

In practical situations we usually have neither a measure nor a set, but rather a number of points. Although there have been some methods of estimating dimension of measures or sets with the use of nearest-point distances (see 6, 11]), they usually work properly only for the case of self-similar (exact-dimensional) measures. Badii and Politi in 2, 3, proposed a dimension function similar to $\operatorname{dim}_{B P}$, which in practice may be estimated in the very same way. Proposition 1.6] specifies rigorously what one obtains by estimating the slope of the log-log graph of the average number of points versus the mean nearest-neighbour distance. Theorem 1.7 says that the obtained value is a bound for the local dimension of measures, while Theorem 2.2 shows its relation to the commonly used Rényi spectrum of dimensions.

If we assume that $\underline{\operatorname{dim}}_{B P}(\mu, q)=\overline{\operatorname{dim}}_{B P}(\mu, q)$ and that they are continuous in $q=0$, then a special case of Theorem 2.2 gives

$$
\operatorname{dim}_{B P}\left(\operatorname{dim}_{R}(\mu, 1)\right)=\operatorname{dim}_{R}(\mu, 1),
$$

where $\operatorname{dim}_{R}(\mu, 1)$ is an important quantity called information dimension, which is equal to capacity (or fractal dimension) for self-similar measures. The relation (4.1) was suggested by Badii and Politi in $[3$ and is used as an estimator for capacity in many papers from various areas of science; a small sample is $[15,1,8,16$. 


\section{REFERENCES}

1. P. Asvestas, S. Golemati, G. K. Matsopoulos, K. S. Nikita, and A. N. Nicolaides, Fractal dimension estimation of carotid atherosclerotic plaques from B-mode ultrasound: a pilot study, Ultrasound in Medicine and Biology 28 (2002), no. 9, 1129-1136.

2. R. Badii and A. Politi, Hausdorff dimension and uniformity factor of strange attractors, Phys. Rev. Lett. 52 (1984), no. 19, 1661-1664. MR741988 (85m:58120)

3. Statistical description of chaotic attractors: the dimension function, J. Statist. Phys. 40 (1985), no. 5-6, 725-750. MR806722 (87c:58067)

4. H. Bauer, Measure and integration theory, de Gruyter Studies in Mathematics, vol. 26, Walter de Gruyter \& Co., Berlin, 2001, translated from the German by Robert B. Burckel. MR.1897176 (2003a:28001)

5. P.J. Clark and F.C. Evans, Distance to nearest neighbor as a measure of spatial relationships in populations, Ecology 35 (1954), 445-453.

6. C. D. Cutler and D. A. Dawson, Estimation of dimension for spatially distributed data and related limit theorems, J. Multivar. Anal. 28 (1989), no. 1, 115-148. MR996987 (90m:62116)

7. D. J. Daley and D. Vere-Jones, An introduction to the theory of point processes. Vol. I, Elementary theory and methods, second ed., Probability and its Applications (New York), Springer-Verlag, New York, 2003. MR.1950431 (2004c:60001)

8. S. G. De Bartolo, M. Veltri, and L. Primavera, Estimated generalized dimensions of river networks, Journal of Hydrology 322 (2006), no. 1-4, 181-191.

9. K. Falconer, Fractal geometry. second edition, John Wiley and Sons, Chichester, 2003. MR 2118797 (2006b:28001)

10. M. Guysinsky and S. Yaskolko, Coincidence of various dimensions associated with metrics and measures on metric spaces, Discrete Contin. Dynam. Systems 3 (1997), no. 4, 591-603. MR:1465128 (98g:58101)

11. P. A. Henderson, Practical methods in ecology, Wiley-Blackwell, 2003.

12. H. G. E. Hentschel and I. Procaccia, The infinite number of generalized dimensions of fractals and strange attractors, Phys. D 8 (1983), no. 3, 435-444. MR719636 (85a:58064)

13. J. Illian, A. Penttinen, H. Stoyan, and D. Stoyan, Statistical analysis and modelling of spatial point patterns, John Wiley \& Sons Ltd., Chichester, 2008. MR2384630 (2008k:62004)

14. J. F. C. Kingman, Poisson processes, Oxford Studies in Probability, vol. 3, Oxford University Press, New York, 1993. MR.1207584 (94a:60052)

15. E. J. Kostelich and H. L. Swinney, Practical considerations in estimating dimension from time series data, Physica Scripta 40 (1989), no. 3, 436-441.

16. R. Lopes and N. Betrouni, Fractal and multifractal analysis: A review, Medical Image Analysis 13 (2009), no. 4, 634-649.

17. Ya. B. Pesin, On rigorous mathematical definitions of correlation dimension and generalized spectrum for dimensions, Journal of Statistical Physics 71 (1993), no. 3-4, 529-547. MR.1219021 (94d:28008)

18. Dimension theory in dynamical systems, Contemporary views and applications, Chicago Lectures in Mathematics, University of Chicago Press, Chicago, IL, 1997. MR.1489237 (99b:58003)

19. R. Rudnicki, Pointwise dimensions and Rényi dimensions, Proc. Amer. Math. Soc. 130 (2002), no. 7, 1981-1982. MR.1896030 (2003a:28016)

20. D. Stoyan and H. Stoyan, Fractals, random shapes and point fields, John Wiley \& Sons, Chichester, 1994. MR:1297125 (95h:60016)

21. Y. Termonia and Z. Alexandrowicz, Fractal dimension of strange attractors from radius versus size of arbitrary clusters, Phys. Rev. Lett. 51 (1983), no. 14, 1265-1268. MR718464 (84k:58155)

Institute of Mathematics, Polish Academy of Sciences, ul. Bankowa 14, 40-007 Katowice, Poland

E-mail address: r.wieczorek@impan.gov.pl 